\title{
Lie Algebra of Infinitesimal Generators of the Symmetry Group of the Heat Equation
}

\author{
Narmanov Otabek Abdigapparovich \\ Tashkent University of Information Technologies, Tashkent, Uzbekistan \\ Email: otabek.narmanov@mail.ru
}

How to cite this paper: Abdigapparovich, N.O. (2018) Lie Algebra of Infinitesimal Generators of the Symmetry Group of the Heat Equation. Journal of Applied Mathematics and Physics, 6, 373-381. https://doi.org/10.4236/jamp.2018.62035

Received: October 6, 2017

Accepted: February 11, 2018

Published: February 14, 2018

Copyright $\odot 2018$ by author and Scientific Research Publishing Inc. This work is licensed under the Creative Commons Attribution International License (CC BY 4.0).

http://creativecommons.org/licenses/by/4.0/

\begin{abstract}
Last time symmetry methods have been recognized to be of great importance for the study of the differential equations arising in mathematics and physics. The purpose of this paper is to provide some application of Lie groups to heat equation. In this example, we determine Lie algebra of infinitesimal generators of symmetry group of heat equation and construct group-invariant solutions of this equation. The some computational methods are presented so that researchers in other fields can readily learn to use them.
\end{abstract}

\section{Keywords}

Lie Group, Heat Equation, Symmetry Group

\section{Introduction}

Suppose we are given differential equation of order $m$

$$
\Delta\left(x, u^{(m)}\right)=0
$$

from $n$ independent of $x=\left(x_{1}, x_{2}, \cdots, x_{n}\right)$ and $q$ dependent variables $u=\left(u_{1}, u_{2}, \cdots, u_{q}\right) \in R^{q}$

Definition. A group $G$ of transformation acting on an open subset $M$ of the space of independent and dependent variables $X \times R^{q}$, is called the symmetry group of Equation (1) if for each solution $u=f(x)$ of Equation (1) and for $g \in G$ such that $g \circ f$ is defined, then the function $\tilde{u}=g \circ f$ is also a solution of the equation.

Remark. The transformation $g$ takes the graph of a function $u=f(x)$ to some set, which is the graph of some function. We denote this function by $g \circ f$.

One of the advantages of knowing the symmetry group of differential equations is that if we know the solution $u=f(x)$ then, in accordance with the de- 
finition, the function $\tilde{u}=g \circ f$ is also a solution for of any element $g$ of the group $G$, so that we have opportunity to construct a whole family of solutions, exposing a known solution to the action of all possible elements of the group. To find the symmetry group, we "continue" the basic space representing independent and dependent variables to a space that also represents all the various partial derivatives that occur in the equation. For a given smooth function $u=f(x)$, there is an induced function $u^{m}=p r^{m} f(x)$, called the m-extension of $f(x)$, equation of the form $u_{j}^{\alpha}=\partial_{j} f^{\alpha}(x)$, where $\partial_{j} f^{\alpha}(x)$ is the derivative of order $\alpha$ of the function $u=f(x)$. Now we can replace the differential equation $\Delta\left(x, u^{(m)}\right)=0$ by an algebraic equation that is defined by the vanishing of the function, which is the right-hand side of the equation $\Delta\left(x, u^{(m)}\right)=0$ defined on $X \times U^{m}$. A smooth solution of the differential equation $\Delta\left(x, u^{(m)}\right)=0$ is a smooth function $u=f(x)$ and its derivatives $u_{j}^{\alpha}=\partial_{j} f^{\alpha}$ must satisfy the algebraic equation

$$
F\left(x, t, p r^{(m)} u(x)\right)=0
$$

The procedure for finding the infinitesimal generators of the symmetry group of differential equations is described in the paper [1]. This procedure uses the prolongation of the action of the symmetry group on the extended space. Infinitesimal generators of the prolongation of the action of the symmetry group are extensions of the infinitesimal generators of the symmetry group of the basic space. We use this scheme to find the symmetry group of the one-dimensional heat equation.

We recall the notion of the algebra of infinitesimal generators of symmetry group. Let $M$ be a smooth manifold of dimension $n, V(M)$-the set of all smooth vector fields on the manifold $M$. For a vector field $X$, let $t \rightarrow X^{t}(x)$ denote the integral curve of a vector field $X$, passing through a point $x \in M$ at $t=0$. The map $t \rightarrow X^{t}(x)$ is defined in some domain $I(x)$, which in the general case does not depend only from the field $X$, but also from the starting point $x$. Further everywhere in formulas of the form $X^{t}(x)$ we assume that $t \in I(x)$. Family $G=\left\{X^{t}: t \in I(x)\right\}$ forms a one-parameter group of transformations of the manifold $M$. Vector field $X$ is called the infinitesimal generators of the transformations group $G$. If the transformation group is $k$-parametric, then is has $k$ infinitesimal generators.

The set $V(M)$ of all smooth vector fields on a manifold is linear space over the field of real numbers and is a Lie algebra with respect to the Lie bracket of vector fields. In general, this algebra is infinite-dimensional Lie algebra.

In this paper we find the Lie algebra of infinitesimal generators of the symmetry group of heat equation. This algebra is a three-dimensional sub algebra of the algebra $V(M)$, where $M$-the space of independent variables. This will allow us to find the complete symmetry group of the heat equation with any heat conduction function $k(u)$ and with the help of these groups, to reduce that heat equation to an ordinary differential equation of the second order. For some heat conduction functions, these equation are studied numerically. 


\section{Main Part}

Let us consider the quasi linear heat equation with coefficient of nonlinearity $k(u)$, which describes the process transfer of heat under the assumption that the medium is stationary and additional sources or sinks of energy in the medium are absent. We consider the following one-dimensional heat equation

$$
u_{t}=\left(k(u) u_{x}\right)_{x}
$$

Finding of infinitesimal generators of the prolongation of the action of the symmetry group is very complicated technically. By this reason first of all we consider a particular case of Equation (3) for $k(u)=u$ :

$$
u_{t}=\left(u u_{x}\right)_{x}
$$

The vector field $X$, which is an infinitesimal generator for the symmetry group of Equation (4) we will seek in the form

$$
X=\tau \frac{\partial}{\partial t}+\xi \frac{\partial}{\partial x}+\varphi \frac{\partial}{\partial u}
$$

The second prolongation $\tilde{X}$ of the vector field $X$ to the extended space of the variables $\left(t, x, u, u_{t}, u_{x}, u_{x x}\right)$ will have the form:

$$
\tilde{X}=X+\varphi^{t} \frac{\partial}{\partial u_{t}}+\varphi^{x} \frac{\partial}{\partial u_{x}}+\varphi^{x x} \frac{\partial}{\partial u_{x x}}
$$

Let $G$ a group of transformations acting on a manifold $M$. A function $F: M \rightarrow N$ where $N$ is a some manifold, is called a $G$-invariant function if for all $x \in M$ and all $g \in G, x \in M$ such that $g(x)$ is defined $F(x)=F(g(x))$. A real-valued $G$-invariant function $F: M \rightarrow R$ is simply called a invariant of $G$. Note that $F: M \rightarrow R^{m}$ is $G$-invariant if and only if each component $F_{I}$ of $F=\left[F_{1}, \cdots, F_{m}\right]$ is an invariant of $G$. If $F: M \rightarrow R^{m}$ is a $G$-invariant function, then clearly every level set of $F$ is a $G$-invariant subset of $M$. However, it is not true that if the set of zeros of a smooth function, $x: F(x)=0$, is an invariant subset of $M$ then the function itself is invariant. However, if every level set of $F$ is invariant function then $F$ is an invariant function. If $G$ acts on $M$ and $F: M \rightarrow R^{m}$ is smooth function, then $F$ is a $G$-invariant function if and only if every level set $F(x)=c, c \in R^{m}$, is a $G$-invariant subset of $M$. It holds the following theorem [1]:

Theorem 1. A real-valued smooth function $F$ is a $G$-invariant function if and only if $\tilde{X}(F)=0$ for every infinitesimal generator $\tilde{X}$ of group $G$.

We will use this theorem-1 for finding of symmetry group of Equation (3). In the space $\left(t, x, u, u_{t}, u_{x}, u_{x x}\right)$, Equation (4) has the form:

$$
F\left(t, x, u, u_{t}, u_{x}, u_{x x}\right)=0 \text {, }
$$

where $F=u_{t}-u_{x}^{2}-u u_{x x}$. Taking into account that $\tilde{X}(F)=0$ we get

$$
-u_{x x} \varphi+\varphi^{t}-2 u_{x} \varphi^{x}-u \varphi^{x x}=0
$$

For the components of the second prolongation $\tilde{X}$ of a vector field $X$, we use their expressions found in [5]: 


$$
\begin{gathered}
\varphi^{x}=\varphi_{x}+\left(\varphi_{u}-\xi_{x}\right) u_{x}-\tau_{x} u_{t}-\xi_{u} u_{x}^{2}-\tau_{u} u_{x} u_{t}, \\
\varphi^{t}=\varphi_{t}-\xi_{t} u_{x}+\left(\varphi_{u}-\tau_{t}\right) u_{t}-\xi_{u} u_{x} u_{t}-\tau_{u} u_{t}^{2}, \\
\varphi^{x x}=\varphi_{x x}+\left(2 \varphi_{x u}-\xi_{x x}\right) u_{x}-\tau_{x x} u_{t}+\left(\varphi_{u u}-3 \xi_{x u}\right) u_{x}^{2}-2 \tau_{x u} u_{x} u_{t}-\xi_{u u} u_{x}^{3} \\
-\tau_{u u} u_{x}^{2} u_{t}+\left(\phi_{u}-2 \xi_{x}\right) u_{x x}-2 \tau_{x} u_{x t}-3 \xi_{u} u_{u} u_{x x}-\tau_{u} u_{x} u_{x x}-2 \tau_{u} u_{x} u_{x t}
\end{gathered}
$$

Substituting these expressions into (8) and taking into account

$$
u_{t}=u u_{x x x}+u_{x}^{2}
$$

We obtain a polynomial on the left-hand side of Equation (8) with respect to the variables $u, u_{x}, u_{x x}, u_{x t}$ :

$$
\begin{aligned}
& -u_{x x} \varphi+\varphi_{t}-\xi_{t} u_{x}+\left(\varphi_{u}-\tau_{t}\right)\left(u_{x}^{2}+u u_{x x}\right)-\xi_{u} u_{x}\left(u_{x}^{2}+u u_{x x}\right)-\tau_{u} u_{t}^{2} \\
& -2 u_{x}\left[\varphi_{x}+\left(\varphi_{u}-\xi_{x}\right) u_{x}-\tau_{x}\left(u_{x}^{2}+u u_{x x}\right)-\xi_{u} u_{x}^{2}-\tau_{u} u_{x}\left(u_{x}^{2}+u u_{x x}\right)\right] \\
& -u\left[\phi_{x x}+\left(2 \phi_{x u}-\xi_{x x}\right) u_{x}-\tau_{x x}\left(u_{x}^{2}+u u_{x x}\right)+\left(\varphi_{u u}-2 \xi_{x x}\right) u_{x}^{2}-2 \tau_{x u} u_{x}\left(u_{x}^{2}+4 u_{x x}\right)\right. \\
& \left.-\xi_{u} u_{x}^{3}-\tau_{u u} u_{x}^{2}\left(u_{x}^{2}+u u_{x x}\right)+\left(\varphi_{u}-2 \xi_{x}\right) u_{x x}-2 \tau_{x} u_{x t}-3 \xi_{u} u_{x} u_{x x}-2 \tau_{u} u_{x} u_{x t}\right]=0
\end{aligned}
$$

Equating the coefficients for different monomials to zero, we obtain the following defining equations for the symmetry group of the heat equation.

From the defining Equation (5) of the Table 1, we obtain that the equality $\varphi=0$ holds. From Equations (11) and (12) we obtain that $\tau_{x}=0, \tau_{u}=0$; the function $\tau$ depends only on $t, \tau=\tau(t)$. From Equation (10) we obtain that $\xi_{u}=0$. From the Equations (9) we obtain that $\tau_{t}=2 \xi_{x}$. From Equation (3) we get that the function $\xi$ does not depend on the variable $t$, that is $\xi_{t}=0$. Therefore, $\xi=\xi(x)$. Equation (6) shows that $\xi_{x x}=0$ i.e. $\xi$ is a linear function of $x: \xi=a x+b$. Since $\tau_{t}=2 \xi_{x}$ takes place, we obtain that $\tau_{t}=2 a$ i.e. $\tau=\tau(t)=2 a t+d$.

Thus we have

$$
X=(a x+b) \frac{\partial}{\partial x}+(2 a t+d) \frac{\partial}{\partial t},
$$

where $a, b, d$-are arbitrary constants. Thus, the Lie algebra of infinitesimal generators of the group of symmetries of the equations is generated by the following vector fields

$$
X_{1}=\frac{\partial}{\partial t}, \quad X_{2}=\frac{\partial}{\partial x}, \quad X_{3}=2 t \frac{\partial}{\partial t}+x \frac{\partial}{\partial x} .
$$

It can be verified that for the Lie brackets of these vector fields it holds equalities

$$
\left[X_{1}, X_{2}\right]=0,\left[X_{1}, X_{2}\right]=2 \frac{\partial}{\partial t},\left[X_{2}, X_{3}\right]=\frac{\partial}{\partial x} .
$$

Now we can prove that in fact Lie algebra of infinitesimal generators of the group of symmetries of the Equations (4) is Lie algebra of infinitesimal generators of the group of symmetries of the Equation (3). 
Table 1. Table of defining equations.

\begin{tabular}{ccc}
\hline No. & Monomial & Defining equation \\
\hline 1 & 1 & $\varphi_{t}=0$ \\
2 & $u$ & $\varphi_{x x}=0$ \\
3 & $u_{x}$ & $-\xi_{t}-2 \varphi_{x}=0$ \\
4 & $u_{x}^{2}$ & $\varphi_{u}-\tau_{t}-2 \varphi_{u}+2 \xi_{x}=0$ \\
5 & $u_{x x}$ & $-\varphi=0$ \\
6 & $u u_{x}$ & $\xi_{x x}-2 \varphi_{x u}=0$ \\
7 & $u_{x} u_{x x}$ & $-\varphi_{u}+2 \xi_{x}+\varphi_{u}-\tau_{t}=0$ \\
8 & $u_{x x}^{2}$ & $-\tau_{u}+\tau_{u}=0$ \\
9 & $u u_{x x}$ & $-\varphi_{u}+2 \xi_{x}+\varphi_{u}-\tau_{t}=0$ \\
10 & $u u_{x} u_{x x}$ & $3 \xi_{u}+2 \tau_{u}=0$ \\
11 & $u u_{x t}$ & $2 \tau_{x}=0$ \\
12 & $u u_{x} u_{x t}$ & $-2 \tau_{u}=0$ \\
13 & $u_{x x} u_{x}^{2}$ & $-\tau_{u u}=0$ \\
\hline
\end{tabular}

Taking into account that $\xi=a x+b, \tau=\tau(t)=2 a t+d, \varphi=0$ for the components of the second prolongation $\tilde{X}$ of a vector field $X$, we have got following expressions:

$$
\varphi^{x}=-a u_{x}, \quad \varphi_{t}=-2 a u_{t}, \quad \varphi^{x x}=-2 a u_{x x} .
$$

Consequently, for the second prolongation $\tilde{X}$ of the vector field $X$ to the extended space of the variables $\left(t, x, u, u_{t}, u_{x}, u_{x x}\right)$ we obtain the following expression

$$
\tilde{X}=(a x+b) \frac{\partial}{\partial x}+(2 a t+d) \frac{\partial}{\partial t}-2 a u_{t} \frac{\partial}{\partial u_{x}}-a u_{x} \frac{\partial}{\partial u_{x}}-2 a u_{x x} \frac{\partial}{\partial u_{x x}}
$$

Now we rewrite Equation (3) in following form

$$
u_{t}-k(u) u_{x x}-k^{\prime} u_{x}^{2}=0
$$

and let us consider the function $F: M \rightarrow R$, where $M$-space of variables $\left(t, x, u, u_{t}, u_{x}, u_{x x}\right), F\left(t, x, u, u_{t}, u_{x}, u_{x x}\right)=u_{t}-k(u) u_{x x}-k^{\prime} u_{x}^{2}$.

Now we can check that

$$
\tilde{X}(F)=2 a\left(u_{t}-k(u) u_{x x}-k^{\prime} u_{x}^{2}\right)=0 .
$$

By the theorem-1 above we can state that for every function $k(u)$ following theorem

Theorem 2. Lie algebra of infinitesimal generators of the symmetry group of Equation (3) is a three-dimensional Lie algebra, generated by vector fields

$$
X_{1}=\frac{\partial}{\partial t}, \quad X_{2}=\frac{\partial}{\partial x}, \quad X_{3}=2 t \frac{\partial}{\partial t}+x \frac{\partial}{\partial x} .
$$

For the Equation (3) of greatest interest is the case when the coefficient of the thermal conductivity $k(u)$ is a nonlinear function of temperature $u$. As studies show, the coefficient of thermal conductivity in a sufficiently wide range of pa- 
rameters can be described by a power-law function of temperature ([1]-[6]), that is, it has the form $k=u^{\alpha}$, where $\sigma>0$.

We consider case $k=u^{\alpha}$, where $\sigma>0$.

The group of transformations generated by the vector field $X_{1}=\frac{\partial}{\partial t}$, consists of time translations $(t, x, u) \rightarrow(t+s, x, u)$. The group of transformations generated by the vector field $X_{2}=\frac{\partial}{\partial x}$, consists of parallel translations of $x$.

$$
(t, x, u) \rightarrow(t, x+s, u)
$$

The group of transformations generated by the vector field $X_{3}=2 t \frac{\partial}{\partial t}+x \frac{\partial}{\partial x}$, consists of transformations of the form:

$$
(t, x, u) \rightarrow\left(e^{2 S} t, e^{s} x, u\right)
$$

Therefore, if $u=u(t, x)$ is a solution of the equation, then the functions

$$
v=u(t-s, x), \quad v=u(t, x-s), \quad v=u\left(e^{-2 s} t, e^{-s} x\right)
$$

are also solutions of Equation (4).

Consider the vector field (the case $a=0$ )

$$
X=d \frac{\partial}{\partial t}+b \frac{\partial}{\partial x}
$$

This vector field generates a transformation of the form

$$
(t, x, u) \rightarrow(t+d s, x+b s, u)
$$

The function $F(t, x)=b t-d x$ is an invariant of these transformations, since $X(F)=0$. Therefore, if $\xi=b t-d x, b=d^{2}, u(t, x)=v(\xi)$, then function $u(t, x)=v(\xi)$ is a solution of Equation (3), where the function $v(\xi)$ is a solution of the following ordinary differential equation

$$
v^{\sigma} v^{\prime \prime}+\sigma v^{\sigma-1} v^{\prime 2}-v^{\prime}=0
$$

In this equation, introducing a new function $z(v)=v^{\prime}$, we obtain the following first-order equation for the function $z$

$$
v^{\sigma} z z^{\prime}+\sigma v^{\sigma-1} z^{2}-z=0
$$

If $z \neq 0$, we obtain the following equation

$$
v^{\sigma} z^{\prime}+\sigma v^{\sigma-1} z-1=0
$$

From this equation we find $z=c v^{-\sigma}$, and $v^{\sigma}=\sigma(\xi+$ const $)$.

In the case $\sigma=1$ we have $v=\xi+$ const. The function $v=\xi+$ const only one of solutions of the following ordinary differential equation

$$
v v^{\prime \prime}+v^{\prime 2}-v^{\prime}=0
$$

which corresponds to the case $\sigma=1$. Equation (14) have solutions which are not included into solutions (12). Now we find this solutions.

In this equation, introducing a new function $z(v)=v^{\prime}$, we obtain the following first-order equation for the function $z$ 


$$
v Z z^{\prime}+z^{2}-z=0
$$

If $z \neq 0$, we obtain the following equation

$$
v z^{\prime}+z-1=0
$$

The constant function $z=1$ is a solution of this equation. In this case $v=\sigma(\xi+$ const $)$, which is included into solutions of (12). If $z \neq 1$, then equation (16) is an equation in complete differentials. The general integral of this equation is the function

$$
F(z, v)=v z-v
$$

If $F(z, v)=v z-v=c$, then $z=\frac{c}{v}+1$.

As a result, for the function $v$ we obtain the following first-order differential equation

$$
v^{\prime}=\frac{c+v}{v} .
$$

As a result of integration, we get that

$$
v-c \ln (v+c)=\xi+c_{1}
$$

where $c, c_{1}$ are arbitrary constants.

Let us consider case the case $\sigma=2$. In the case from (12) we obtain $v^{2}=2 \xi+c$, where $c$-integration constant. This function gives all solutions of following ordinary differential equation

$$
v^{2} v^{\prime \prime}+2 v v^{\prime 2}-v^{\prime}=0
$$

Consider infinitesimal generator (the case $a \neq 0$ )

$$
X=2 a t \frac{\partial}{\partial t}+a x \frac{\partial}{\partial x} .
$$

In this case, the function $\xi=\frac{x}{\sqrt{t}}$ is an invariant of the group of transformations generated by the this vector field since $X(\xi)=0$. In this case, the we will seek solution of (3) in the form:

$$
u(t, x)=v(\xi)
$$

where the function $v(\xi)$ is a solution of equation

$$
v^{\sigma} v^{\prime \prime}+\sigma v^{\sigma-1} v^{\prime 2}+\frac{\xi}{2} v^{\prime}=0 .
$$

In the case $\sigma=1$ the Equation (18) has the following form

$$
v v^{\prime \prime}+v^{\prime 2}+\frac{\xi}{2} v^{\prime}=0
$$

By using the Maple-13 software package, its commands "dsolve", "DEplot", "with (DEtools)" we numerically studied the solution of the Equation (19).

Numerical analyst shows that solution of this equation limited for $\xi \rightarrow \infty$ (Figure 1). This means that the temperature is limited. 


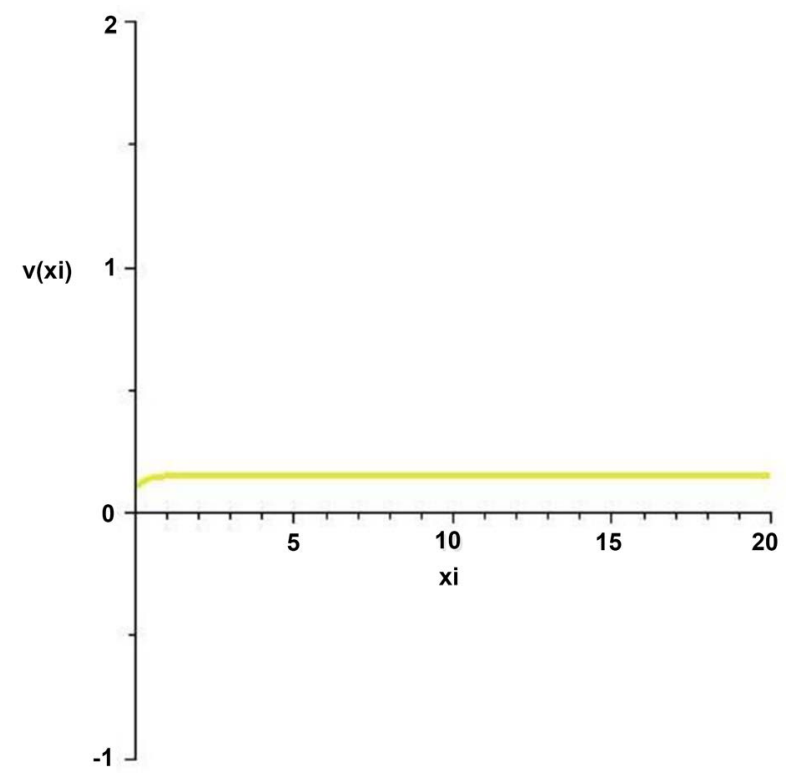

Figure 1. The initial conditions $v(0)=0.1, v^{\prime}(0)=1$.

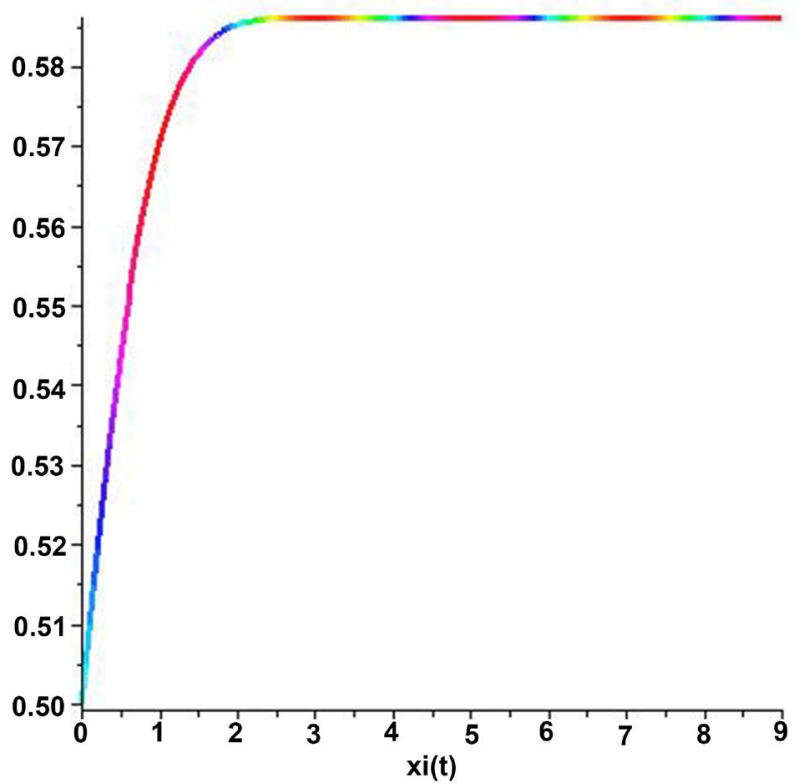

Figure 2. With the initial conditions $v 0.5, v^{\prime}(0)=0.1$.

In the case $\sigma=2$ the Equation (18) has the following form

$$
v^{2} v^{\prime \prime}+2 v v^{\prime 2}+\frac{\xi}{2} v^{\prime}=0
$$

for the function $v(\xi)$.

From this equation we can proceed to the system of differential equations of first order

$$
v^{\prime}=\omega, \quad \omega^{\prime}=-2 \frac{\omega^{2}}{v}-\frac{\xi \omega}{2 v^{2}}
$$


As the case $\sigma=1$ again in the case $\sigma=2$ by investigation of system (21) with numerical methods using the Maple-13 software package, we obtain that the solution of the equation (20) is limited for $\xi \rightarrow \infty$ (Figure 2). This means that in this case also the temperature is limited.

\section{References}

[1] Olver, P.J. (1993) Applications of Lie Groups to Differential Equations. Springer, New York.

[2] Bruno, A.D. (2000) Self-Similar Solution and Power Geometry. Russian Mathematical Surveys, 22, 1-42.

[3] Volosevich, L. (1997) Self-Similar Solutions to the Problems of Gas Dynamics. MFTI, Moscow. (In Russian)

[4] Ibragimov, N.X. (1991) Opit gruppovogo analiza obiknovennix differentialnix uravneniy. Znanie, Moscow. (In Russian)

[5] Bratus, A.S., Novozhilov, A.S. and Platonov, A.P. (2010) Dynamical Systems and Models in Biology. Fizmatlit, Moscow. (In Russian)

[6] Samarskiy, A.A., Galaktionov, V.A., Kurdumov, S.P. and Mikhaylov, A.P. (1987) Rejimi s obostreniyem $\mathrm{v}$ zadachax dlya kvazilinynix parabolicheskix uravneniy. Nauka, Moscow. (In Russian) 